\title{
Some generalized nonlinear retarded integral inequalities with applications
}

Wu-Sheng Wang

Correspondence: wang4896@126. com

Department of Mathematics, Hechi University, Guangxi, Yizhou 546300, P. R. China

\section{Abstract}

In this article we discuss some new generalized nonlinear Gronwall-Bellman-Type integral inequalities with two variables, which include a non-constant term outside the integrals. We use our result to deal with the estimate on the solutions of partial differential equations with the initial and boundary conditions.

Mathematics Subject Classification 2000: 26D10; 26D15; 26D20; 34A40.

Keywords: integral inequality, integral inequality technique, boundary value problem, boundedness, uniqueness

\section{Introduction}

Various generalizations of Gronwall inequality [1,2] are fundamental tools in the study of existence, uniqueness, boundedness, stability and other qualitative properties of solutions of differential equations, integral equations, and differential-integral equations. There are a lot of articles investigating its generalizations such as [3-23]. Recently, Pachpatte [19] provided the explicit estimations of following integral inequalities:

$$
\begin{aligned}
& u^{p}(t) \leq c+p \sum_{i=1}^{n} \int_{\alpha_{i}\left(t_{0}\right)}^{\alpha_{i}(t)}\left[a_{i}(s) u^{p}(s)+b_{i}(s) u(s)\right] d s \\
& u^{p}(t) \leq c+p \sum_{i=1}^{n} \int_{\alpha_{i}\left(t_{0}\right)}^{\alpha_{i}(t)}\left[a_{i}(s) u(s) w(u(s))+b_{i}(s) u(s)\right] d s
\end{aligned}
$$

and

$$
\begin{aligned}
& u^{p}(x, y) \leq c+p \sum_{i=1}^{n} \int_{\alpha_{i}\left(x_{0}\right)}^{\alpha_{i}(x)} \int_{\beta_{i}\left(y_{0}\right)}^{\beta_{i}(y)}\left[a_{i}(s, t) u^{p}(s, t)+b_{i}(s, t) u(s, t)\right] d t d s, \\
& u^{p}(x, y) \leq c+p \sum_{i=1}^{n} \int_{\alpha_{i}\left(x_{0}\right)}^{\alpha_{i}(x)} \int_{\beta_{i}\left(y_{0}\right)}^{\beta_{i}(y)}\left[a_{i}(s, t) u(s, t) w(u(s, t))+b_{i}(s, t) u(s, t)\right] d t d s,
\end{aligned}
$$

\section{空


where $c$ is a constant. Cheung [7] investigated the inequality

$$
\begin{aligned}
u^{p}(x, y) \leq & a+\frac{p}{p-q} \int_{b_{1}\left(x_{0}\right)}^{b_{1}(x)} \int_{c_{1}\left(y_{0}\right)}^{c_{1}(y)} g_{1}(s, t) u^{q}(s, t) d t d s \\
& +\frac{p}{p-q} \int_{b_{2}\left(x_{0}\right)}^{b_{2}(x)} \int_{c_{2}\left(y_{0}\right)}^{c_{2}(y)} g_{2}(s, t) u^{q}(s, t) \psi(u(s, t)) d t d s .
\end{aligned}
$$

Agarwal et al. [3] obtained the explicit bounds to the solutions of the following retarded integral inequalities:

$$
\begin{aligned}
& \varphi(u(t)) \leq c+\sum_{i=1}^{n} \int_{\alpha_{i}\left(t_{0}\right)}^{\alpha_{i}(t)} u^{q}(s)\left[f_{i}(s) \varphi(u(s))+g_{i}(s)\right] d s, \\
& \varphi(u(t)) \leq c+\sum_{i=1}^{n} \int_{\alpha_{i}\left(t_{0}\right)}^{\alpha_{i}(t)} u^{q}(s)\left[f_{i}(s) \varphi_{1}(u(s))+g_{i}(s) \varphi_{2}(\log (u(s)))\right] d s, \\
& \varphi(u(t)) \leq c+\sum_{i=1}^{n} \int_{\alpha_{i}\left(t_{0}\right)}^{\alpha_{i}(t)} u^{q}(s)\left[f_{i}(s) L(s, u(s))+g_{i}(s) u(s)\right] d s,
\end{aligned}
$$

where $c$ is a constant. Chen et al. [6] discussed the following inequalities:

$$
\begin{aligned}
& \psi(u(x, y)) \leq c+\int_{\gamma\left(x_{0}\right)}^{\gamma(x)} \int_{\delta\left(y_{0}\right)}^{\delta(y)} f(s, t) \varphi(u(s, t)) d t d s, \\
& \psi(u(x, y)) \leq c+\int_{\alpha\left(x_{0}\right)}^{\alpha(x)} \int_{\beta\left(y_{0}\right)}^{\beta(y)} g(s, t) u(u, s) d t d s \\
& +\int_{\gamma\left(x_{0}\right)}^{\gamma(x)} \int_{\delta\left(y_{0}\right)}^{\delta(y)} f(s, t) u(s, t) \varphi(u(s, t)) d t d s, \\
& \psi(u(x, y)) \leq c+\int_{\alpha\left(x_{0}\right)}^{\alpha(x)} \int_{\beta\left(y_{0}\right)}^{\beta(y)} g(s, t) w(u(s, t)) d t d s \\
& +\int_{\gamma\left(x_{0}\right)}^{\gamma(x)} \int_{\delta\left(y_{0}\right)}^{\delta(y)} f(s, t) w(u(s, t)) \varphi(u(s, t)) d t d s,
\end{aligned}
$$

where $c$ is a constant.

In this article, motivated mainly by the works of Agarwal et al. [3] and Chen et al. [6], Cheung [7], Pachpatte [19], we discuss more general forms of following integral inequalities:

$$
\begin{aligned}
\psi(u(x, y)) \leq & a(x, y)+b(x, y) \sum_{i=1}^{n} \int_{\alpha_{i}\left(x_{0}\right)}^{\alpha_{i}(x)} \int_{\beta_{i}\left(y_{0}\right)}^{\beta_{i}(y)} w(u(s, t))\left[f_{i}(s, t) \varphi(u(s, t))\right. \\
& \left.+g_{i}(s, t)\right] d t d s,
\end{aligned}
$$




$$
\begin{aligned}
\psi(u(x, y)) \leq & a(x, y)+b(x, y) \sum_{i=1}^{n} \int_{\alpha_{i}\left(x_{0}\right)}^{\alpha_{i}(x)} \int_{\beta_{i}\left(y_{0}\right)}^{\beta_{i}(y)} w(u(s, t))\left[f_{i}(s, t) \varphi_{1}(u(s, t))\right. \\
& \left.+g_{i}(s, t) \varphi_{2}(\log (u(s, t)))\right] d t d s, \\
\psi(u(x, y)) \leq & a(x, y)+b(x, y) \sum_{i=1}^{n} \int_{\alpha_{i}\left(x_{0}\right)}^{\alpha_{i}(x)} \int_{\beta_{i}\left(y_{0}\right)}^{\beta_{i}(y)} w(u(s, t))\left[f_{i}(s, t) L(s, t, u(s, t))\right. \\
& \left.+g_{i}(s, t) u(s, t)\right] d t d s,
\end{aligned}
$$

for $(x, y) \in\left[x_{0}, x_{1}\right) \times\left[y_{0}, y_{1}\right)$, where $a(x, y), b(x, y)$ are nonnegative and nondecreasing functions in each variable. In inequalities (1.1)-(1.3), we generalized the constant $c$ in $[1,5]$ to the function $a(x, y)$, the function $u(x)$ in [1] to the $u(x, y)$ with two variables.

\section{Main result}

Throughout this article, $x_{0}, x_{1}, y_{0}, y_{1} \in \mathbb{R}$ are given numbers. $I:=\left[x_{0}, x_{1}\right), J:=\left[y_{0}, y_{1}\right)$, $\Delta:=\left[x_{0}, x_{1}\right) \times\left[y_{0}, y_{1}\right), \mathbb{R}_{+}:=[0, \infty)$. Consider (1.1)-(1.3), and suppose that

$\left(H_{1}\right) \psi \in C\left(\mathbb{R}_{+}, \mathbb{R}_{+}\right)$is a strictly increasing function with $\psi(0)=0$ and $\psi(t) \rightarrow \infty$ as $t \rightarrow \infty$;

$\left(H_{2}\right) a, b: \Delta \rightarrow(0, \infty)$ are nondecreasing in each variable;

$\left(H_{3}\right) w, \varphi, \varphi_{1}, \varphi_{2} \in C\left(\mathbb{R}_{+}, \mathbb{R}_{+}\right)$are nondecreasing with $w(0)>0, \varphi(r)>0, \varphi_{1}(r)>0$ and $\varphi_{2}(r)>0$ for $r>0$;

$\left(H_{4}\right) \alpha_{i} \in C^{1}(I, I)$ and $\beta_{i} \in C^{1}(J, J)$ are nondecreasing such that $\alpha_{i}(x) \leq x, \alpha_{i}\left(x_{0}\right)=x_{0}, \beta_{i}$ $(y) \leq y$ and $\beta_{i}\left(y_{0}\right)=y_{0}, i=1,2, \ldots, n$;

$\left(H_{5}\right) f_{i}, g_{i} \in C\left(\Delta, \mathbb{R}_{+}\right), i=1,2, \ldots, n$.

Theorem 1. Suppose that $\left(H_{1}-H_{5}\right)$ hold and $u(x, y)$ is a nonnegative and continuous function on $\Delta$ satisfying (1.1). Then we have

$$
u(x, y) \leq \psi^{-1}\left(W^{-1}\left(\Phi^{-1}(B(x, y))\right)\right)
$$

for all $(x, y) \in\left[x_{0}, X_{1}\right) \times\left[y_{0}, Y_{1}\right)$, where

$$
\begin{aligned}
& W(r):=\int_{1}^{r} \frac{d s}{w\left(\psi^{-1}(s)\right)}, \quad r>0, \quad W(0):=\lim _{r \rightarrow 0^{+}} W(r) \\
& \Phi(r):=\int_{1}^{r} \frac{d s}{\varphi\left(\psi^{-1}\left(W^{-1}(s)\right)\right)}, \quad r>0, \quad \Phi(0):=\lim _{r \rightarrow 0^{+}} \Phi(r) \\
& B(x, y):=\Phi(A(x, y))+b(x, y) \sum_{i=1}^{n} \int_{\alpha_{i}\left(x_{0}\right)}^{\alpha_{i}(x)} \int_{\beta_{i}\left(y_{0}\right)}^{\beta_{i}(y)} f_{i}(s, t) d t d s \\
& A(x, y):=W(a(x, y))+b(x, y) \sum_{i=1}^{n} \int_{\alpha_{i}\left(x_{0}\right)}^{\alpha_{i}(x)} \int_{\beta_{i}\left(y_{0}\right)}^{\beta_{i}(y)} g_{i}(s, t) d t d s
\end{aligned}
$$


$\psi^{-1}, W^{-1}$ and $\Phi^{-1}$ denote the inverse function of $\psi, W$ and $\Phi$, respectively, and $\left(X_{1}, Y_{1}\right)$ $\in \Delta$ is arbitrarily given on the boundary of the planar region

$$
\mathcal{R}:=\left\{(x, y) \in \Delta: B(x, y) \in \operatorname{Dom}\left(\Phi^{-1}\right), \Phi^{-1}(B(x, y)) \in \operatorname{Dom}\left(W^{-1}\right)\right\} .
$$

Proof. From assumption $\mathrm{H}_{2}$ and the inequality (1.1), we have

$$
\psi(u(x, y)) \leq a(X, y)+b(X, y) \sum_{i=1}^{n} \int_{\alpha_{i}\left(x_{0}\right)}^{\alpha_{i}(x)} \int_{\beta_{i}\left(y_{0}\right)}^{\beta_{i}(y)} w(u(s, t))\left[f_{i}(s, t) \varphi(u(s, t))+g_{i}(s, t)\right] d t d s,
$$

for all $(x, y) \in\left[x_{0}, X\right] \times\left[y_{0}, y_{1}\right)$, where $x_{0} \leq X \leq X_{1}$ is chosen arbitrarily. Define a function $\eta(x, y)$ by the right-hand side of (2.7). Clearly, $\eta(x, y)$ is a positive and nondecreasing function in each variable, $\eta\left(x_{0}, y\right)=a(X, y)>0$. Then, (2.7) is equivalent to

$$
u(x, y) \leq \psi^{-1}(\eta(x, y))
$$

for all $(x, y) \in\left[x_{0}, X\right] \times\left[y_{0}, y_{1}\right)$. By the fact that $\alpha_{i}(x) \leq x$ for $x \in\left[x_{0}, x_{1}\right), \beta_{i}(y) \leq y$ for $y$ $\in\left[y_{0}, y_{1}\right), i=1,2, \ldots, n$, and the monotonicity of $w, \psi^{-1}, \eta$, we have for all $(x, y) \in\left[x_{0}, X\right] \times$ $\left[y_{0}, y_{1}\right)$,

$$
\begin{aligned}
\eta_{x}(x, y)= & b(X, y) \sum_{i=1}^{n} \alpha^{\prime}(x) \int_{\beta_{i}\left(y_{0}\right)}^{\beta_{i}(y)} w\left(u\left(\alpha_{i}(x), t\right)\right)\left[f_{i}\left(\alpha_{i}(x), t\right) \varphi\left(u\left(\alpha_{i}(x), t\right)\right)+g_{i}\left(\alpha_{i}(x), t\right)\right] d t \\
\leq & w\left(\psi^{-1}(\eta(x, y))\right) b(X, y) \sum_{i=1}^{n} \alpha_{i}^{\prime}(x) \int_{\beta_{i}\left(y_{0}\right)}^{\beta_{i}(y)}\left[f_{i}\left(\alpha_{i}(x), t\right) \varphi\left(\psi^{-1}\left(\eta\left(\alpha_{i}(x), t\right)\right)\right)\right] \\
& \left.+g_{i}\left(\alpha_{i}(x), t\right)\right] d t .
\end{aligned}
$$

From (2.9), we get

$$
\begin{aligned}
\frac{\eta_{x}(x, y)}{w\left(\psi^{-1}(\eta(x, y))\right)} \leq & b(X, y) \sum_{i=1}^{n} \alpha^{\prime}{ }_{i}(x) \int_{\beta_{i}\left(\gamma_{0}\right)}^{\beta_{i}(y)}\left[f_{i}\left(\alpha_{i}(x), t\right) \varphi\left(\psi^{-1}\left(\eta\left(\alpha_{i}(x), t\right)\right)\right)\right. \\
& \left.+g_{i}\left(\alpha_{i}(x), t\right)\right] d t
\end{aligned}
$$

for all $(x, y) \in\left[x_{0}, X\right] \times\left[y_{0}, y_{1}\right)$. Integrating (2.10) from $x_{0}$ to $x$, by the definition of $W$ in (2.2), we get for all $(x, y) \in\left[x_{0}, X\right] \times\left[y_{0}, y_{1}\right)$,

$$
\begin{aligned}
W(\eta(x, y)) \leq & W\left(\eta\left(x_{0}, y\right)\right)+b(X, \gamma) \sum_{i=1}^{n} \int_{\alpha_{i}\left(x_{0}\right)}^{\alpha_{i}(x)} \int_{\beta_{i}\left(y_{0}\right)}^{\beta_{i}(y)}\left[f_{i}(s, t) \varphi\left(\psi^{-1}(\eta(s, t))\right)+g_{i}(s, t)\right] d t d s \\
= & W(a(X, y))+b(X, y) \sum_{i=1}^{n} \int_{\alpha_{i}\left(x_{0}\right)}^{\alpha_{i}(x)} \int_{\beta_{i}\left(y_{0}\right)}^{\beta_{i}(y)}\left[f_{i}(s, t) \varphi\left(\psi^{-1}(\eta(s, t))\right)+g_{i}(s, t)\right] d t d s \\
\leq & W(a(X, \gamma))+b(X, \gamma) \sum_{i=1}^{n} \int_{\alpha_{i}\left(x_{0}\right)}^{\alpha_{i}(X)} \int_{\beta_{i}\left(y_{0}\right)}^{\beta_{i}(y)} \beta_{i}(s, t) d t d s \\
& +b(X, Y) \sum_{i=1}^{n} \int_{\alpha_{i}\left(x_{0}\right)}^{\alpha_{i}(x)} \int_{\beta_{i}\left(y_{0}\right)}^{\beta_{i}(y)} f_{i}(s, t) \varphi\left(\psi^{-1}(\eta(s, t))\right) d t d s \\
= & c(X, \gamma)+b(X, y) \sum_{i=1}^{n} \int_{\alpha_{i}\left(x_{0}\right)}^{\alpha_{i}(x)} \int_{\beta_{i}\left(y_{0}\right)}^{\beta_{i}(y)} f_{i}(s, t) \varphi\left(\psi^{-1}(\eta(s, t))\right) d t d s,
\end{aligned}
$$


where

$$
c(X, y)=W(a(X, y))+b(X, y) \sum_{i=1}^{n} \int_{\alpha_{i}\left(x_{0}\right)}^{\alpha_{i}(x)} \int_{\beta_{i}\left(y_{0}\right)}^{\beta_{i}(y)} g_{i}(s, t) d t d s .
$$

Now, define a function $\Gamma(x, y)$ by the right-hand side of (2.11). Clearly, $\Gamma(x, y)$ is a positive and nondecreasing function in each variable, $\Gamma\left(x_{0}, y\right)=c(X, y)>0$. then, (2.11) is equivalent to

$$
\eta(x, y) \leq W^{-1}(\Gamma(x, y))
$$

for all $(x, y) \in\left[x_{0}, X\right] \times\left[y_{0}, Y_{1}\right)$, where $Y_{1}$ is defined in (2.6). By the fact that $\alpha_{i}(x) \leq x$ for $x \in\left[x_{0}, x_{1}\right), \beta_{i}(y) \leq y$ for $y \in\left[y_{0}, y_{1}\right), i=1,2, \ldots, n$, and the monotonicity of $\varphi, \psi^{-1}, W$ ${ }^{1}, \Gamma$, we have for all $(x, y) \in\left[x_{0}, X\right] \times\left[y_{0}, Y_{1}\right)$,

$$
\begin{aligned}
\Gamma_{x}(x, y) & =b(X, y) \sum_{i=1}^{n} \alpha_{i}^{\prime}(x) \int_{\beta_{i}\left(y_{0}\right)}^{\beta_{i}(y)} f_{i}\left(\alpha_{i}(x), t\right) \varphi\left(\psi^{-1}\left(\eta\left(\alpha_{i}(x), t\right)\right)\right) d t \\
& \leq b(X, y) \varphi\left(\psi^{-1}\left(W^{-1}(\Gamma(x, y))\right)\right) \sum_{i=1}^{n} \alpha_{i}{ }^{\prime}(x) \int_{\beta_{i}\left(y_{0}\right)}^{\beta_{i}(y)} f_{i}\left(\alpha_{i}(x), t\right) d t .
\end{aligned}
$$

From (2.14), we have for all $(x, y) \in\left[x_{0}, X\right] \times\left[y_{0}, Y_{1}\right)$,

$$
\frac{\Gamma_{x}(x, y)}{\varphi\left(\psi^{-1}\left(W^{-1}(\Gamma(x, y))\right)\right)} \leq b(X, y) \sum_{i=1}^{n} \alpha_{i}{ }^{\prime}(x) \int_{\beta_{i}\left(y_{0}\right)}^{\beta_{i}(y)} f_{i}\left(\alpha_{i}(x), t\right) d t .
$$

Integrating (2.15) from $x_{0}$ to $x$, by the definition of $\Phi$ in (2.3), we get

$$
\begin{aligned}
\Phi(\Gamma(x, y)) & \leq \Phi\left(\Gamma\left(x_{0} y\right)\right)+b(X, y) \sum_{i=1}^{n} \int_{\alpha_{i}\left(x_{0}\right)}^{\alpha_{i}(x)} \int_{\beta_{i}\left(y_{0}\right)}^{\beta_{i}(y)} f_{i}(s, t) d t d s \\
& =\Phi(c(X, Y))+b(X, y) \sum_{i=1}^{n} \int_{\alpha_{i}\left(x_{0}\right)}^{\alpha_{i}(x)} \int_{\beta_{i}\left(y_{0}\right)}^{\beta_{i}(y)} f_{i}(s, t) d t d s,
\end{aligned}
$$

for all $(x, y) \in\left[x_{0}, X\right] \times\left[y_{0}, Y_{1}\right)$. From (2.12) and (2.16), we find

$$
\begin{aligned}
\Gamma(x, y) \leq & \Phi^{-1}\left(\Phi(c(X, y))+b(X, y) \sum_{i=1}^{n} \int_{\alpha_{i}\left(x_{0}\right)}^{\alpha_{i}(x)} \int_{\beta_{i}\left(y_{0}\right)}^{\beta_{i}(y)} f_{i}(s, t) d t d s\right) \\
= & \Phi^{-1}\left(\Phi\left(W(a(X, y))+b(X, y) \sum_{i=1}^{n} \int_{\alpha_{i}\left(x_{0}\right)}^{\alpha_{i}(X)} \int_{\beta_{i}\left(y_{0}\right)}^{\beta_{i}(y)} g_{i}(s, t) d t d s\right)\right. \\
& \left.+b(X, y) \sum_{i=1}^{n} \int_{\alpha_{i}\left(x_{0}\right)}^{\alpha_{i}(x)} \int_{\beta_{i}\left(y_{0}\right)}^{\beta_{i}(y)} f_{i}(s, t) d t d s\right)
\end{aligned}
$$


for all $(x, y) \in\left[x_{0}, X\right] \times\left[y_{0}, Y_{1}\right)$. From (2.8), (2.13), and (2.17), we get

$$
\begin{aligned}
u(x, y) \leq & \psi^{-1}(\eta(x, y)) \leq \psi^{-1}\left(W^{-1}(\Gamma(x, y))\right) \\
\leq & \psi^{-1}\left(W ^ { - 1 } \left(\Phi ^ { - 1 } \left(\Phi\left(W(a(X, y))+b(X, y) \sum_{i=1}^{n} \int_{\alpha_{i}\left(x_{0}\right)}^{\alpha_{i}(X)} \int_{\beta_{i}\left(y_{0}\right)}^{\beta_{i}(y)} g_{i}(s, t) d t d s\right)\right.\right.\right. \\
& \left.\left.+b(X, y) \sum_{i=1}^{n} \int_{\alpha_{i}\left(x_{0}\right)}^{\alpha_{i}(x)} \int_{\beta_{i}\left(y_{0}\right)}^{\beta_{i}(y)} f_{i}(s, t) d t d s\right)\right),
\end{aligned}
$$

for all $(x, y) \in\left[x_{0}, X\right] \times\left[y_{0}, Y_{1}\right)$. Let $x=X$, from (2.18), we observe that

$$
\begin{aligned}
u(X, \gamma) \leq & \psi^{-1}\left(W ^ { - 1 } \left(\Phi ^ { - 1 } \left(\Phi\left(W(a(X, y))+b(X, \gamma) \sum_{i=1}^{n} \int_{\alpha_{i}\left(x_{0}\right)}^{\alpha_{i}(X)} \int_{\beta_{i}\left(y_{0}\right)}^{\beta_{i}(y)} g_{i}(s, t) d t d s \mid\right)\right.\right.\right. \\
& \left.\left.\left.+b(X, \gamma) \sum_{i=1}^{n} \int_{\alpha_{i}\left(x_{0}\right)}^{\alpha_{i}(x)} \int_{\beta_{i}\left(y_{0}\right)}^{\beta_{i}(y)} f_{i}(s, t) d t d s\right)\right)\right)
\end{aligned}
$$

for all $(X, y) \in\left[x_{0}, X_{1}\right) \times\left[y_{0}, Y_{1}\right)$, where $X_{1}$ is defined by (2.6). Since $X \in\left[x_{0}, X_{1}\right)$ is arbitrary, from (2.19), we get the required estimations

$$
\begin{aligned}
u(x, y) \leq & \psi^{-1}\left(W ^ { - 1 } \left(\Phi ^ { - 1 } \left(\Phi\left(W(a(x, y))+b(x, y) \sum_{i=1}^{n} \int_{\alpha_{i}\left(x_{0}\right)}^{\alpha_{i}(X)} \int_{\beta_{i}\left(y_{0}\right)}^{\beta_{i}(y)} g_{i}(s, t) d t d s\right)\right.\right.\right. \\
& \left.\left.+b(x, y) \sum_{i=1}^{n} \int_{\alpha_{i}\left(x_{0}\right)}^{\alpha_{i}(x)} \int_{\beta_{i}\left(y_{0}\right)}^{\beta_{i}(y)} f_{i}(s, t) d t d s\right)\right),
\end{aligned}
$$

for all $(x, y) \in\left[x_{0}, X_{1}\right) \times\left[y_{0}, Y_{1}\right)$. Theorem 1 is proved.

Remark that Theorem 1 generalizes Theorem 2.1 in [3].

Theorem 2. Suppose that $\left(H_{1}-H_{5}\right)$ hold and $u(x, y)$ is a nonnegative and continuous function on $\Delta$ satisfying (1.2). Then

(i) if $\varphi_{1}(u) \geq \varphi_{2}(\log (u))$, we have

$$
u(x, y) \leq \psi^{-1}\left[W^{-1}\left(\psi_{1}^{-1}\left(D_{1}(x, y)\right)\right)\right],
$$

for all $(x, y) \in\left[x_{0}, X_{2}\right) \times\left[y_{0}, Y_{2}\right)$,

(ii) if $\varphi_{1}(u)<\varphi_{2}(\log (u))$, we have

$$
u(x, y) \leq \psi^{-1}\left[W^{-1}\left(\Psi_{2}^{-1}\left(D_{2}(x, y)\right)\right)\right],
$$

for all $(x, y) \in\left[x_{0}, X_{3}\right) \times\left[y_{0}, Y_{3}\right)$, where $W$ is defined by (2.2) in Theorem 1 ,

$$
\begin{aligned}
D_{j}(x, y) & :=\Psi_{j}(W(a(x, y)))+b(x, y) \sum_{i=1}^{n} \int_{\alpha_{i}\left(x_{0}\right)}^{\alpha_{i}(x)} \int_{\beta_{i}\left(y_{0}\right)}^{\beta_{i}(y)}\left[f_{i}(s, t)+g_{i}(s, t)\right] d t d s, \\
\Psi_{j}(r) & :=\int_{1}^{r} \frac{d s}{\varphi_{j}\left(\psi^{-1}\left(W^{-1}(s)\right)\right)}, \quad \Psi_{j}(0):=\lim _{r \rightarrow 0+} \Psi_{j}(r),
\end{aligned}
$$


$j=1,2, \psi^{-1}, W^{-1}, \Psi_{1}^{-1}$ and $\Psi_{2}^{-1}$ denote the inverse function of $\psi, W, \Psi_{1}$ and $\Psi_{2}$, respectively, $\left(X_{2}, Y_{2}\right)$ is arbitrarily given on the boundary of the planar region

$$
\mathcal{R}_{1}:=\left\{(x, y) \in \Delta: D_{1}(x, y) \in \operatorname{Dom}\left(\Psi_{1}^{-1}\right), \Psi_{1}^{-1}\left(D_{1}(x, y)\right) \in \operatorname{Dom}\left(W^{-1}\right)\right\}
$$

and $\left(X_{3}, Y_{3}\right)$ is arbitrarily given on the boundary of the planar region

$$
\mathcal{R}_{2}:=\left\{(x, y) \in \Delta: D_{2}(x, y) \in \operatorname{Dom}\left(\Psi_{2}^{-1}\right), \Psi_{2}^{-1}\left(D_{2}(x, y)\right) \in \operatorname{Dom}\left(W^{-1}\right)\right\} .
$$

Proof. From the inequality (1.2), we have

$$
\begin{gathered}
\psi(u(x, y)) \leq a(X, y)+b(X, y) \sum_{i=1}^{n} \int_{\alpha_{i}\left(x_{0}\right)}^{\alpha_{i}(x)} \int_{\beta_{i}\left(y_{0}\right)}^{\beta_{i}(y)} w(u(s, t))\left[f_{i}(s, t) \varphi_{1}(u(s, t))\right. \\
\left.+g_{i}(s, t) \varphi_{2}(\log (u(s, t)))\right] d t d s,
\end{gathered}
$$

for all $(x, y) \in\left[x_{0}, X\right] \times\left[y_{0}, y_{1}\right)$, where $x_{0} \leq X \leq X_{2}$ is chosen arbitrarily. Let $\Xi(x, y)$ denote the right-hand side of (2.25), which is a positive and nondecreasing function in each variable, $\Xi\left(x_{0}, y\right)=a(X, y)$. Then, (2.25) is equivalent to $u(x, y) \leq \psi^{-1}(\Xi(x, y))$. By the fact that $\alpha_{i}(x) \leq x$ for $x \in\left[x_{0}, x_{1}\right), \beta_{i}(y) \leq y$ for $y \in\left[y_{0}, y_{1}\right), i=1,2, \ldots, n$, and the monotonicity of $w, \psi^{-1}, \Xi$, we have for all $(x, y) \in\left[x_{0}, X\right] \times\left[y_{0}, y_{1}\right)$,

$$
\begin{aligned}
\Xi_{x}(x, y)= & b(X, y) \sum_{i=1}^{n} \alpha_{i}{ }^{\prime}(x) \int_{\beta_{i}\left(y_{0}\right)}^{\beta_{i}(y)} w\left(u\left(\alpha_{i}(s), t\right)\right)\left[f_{i}\left(\alpha_{i}(x), t\right) \varphi_{1}\left(u\left(\alpha_{i}(x), t\right)\right)\right. \\
& \left.+g_{i}\left(\alpha_{i}(x), t\right) \varphi_{2}\left(\log \left(u\left(\alpha_{i}(x), t\right)\right)\right)\right] d t \\
\leq & b(X, \gamma) w\left(\psi^{-1}(\Xi(x, y))\right) \sum_{i=1}^{n} \alpha_{i}{ }^{\prime}(x) \int_{\beta_{i}\left(y_{0}\right)}^{\beta_{i}(y)}\left[f_{i}\left(\alpha_{i}(x), t\right) \varphi_{1}\left(\psi^{-1}\left(\Xi\left(\alpha_{i}(x), t\right)\right)\right)\right. \\
& \left.+g_{i}\left(\alpha_{i}(x), t\right) \varphi_{2}\left(\log \left(\psi^{-1}\left(\Xi\left(\alpha_{i}(x), t\right)\right)\right)\right)\right] d t
\end{aligned}
$$

for all $(x, y) \in\left[x_{0}, X\right] \times\left[y_{0}, y_{1}\right)$. From (2.26), we have

$$
\begin{aligned}
\frac{\Xi_{x}(x, y)}{w\left(\psi^{-1}(\Xi(x, y))\right)} \leq & b(X, \gamma) \sum_{i=1}^{n} \alpha_{i}{ }^{\prime}(x) \int_{\beta_{i}\left(y_{0}\right)}^{\beta_{i}(y)}\left[f_{i}\left(\alpha_{i}(x), t\right) \varphi_{1}\left(\psi^{-1}\left(\Xi\left(\alpha_{i}(x), t\right)\right)\right)\right. \\
& \left.+g_{i}\left(\alpha_{i}(x), t\right) \varphi_{2}\left(\log \left(\psi^{-1}\left(\Xi\left(\alpha_{i}(x), t\right)\right)\right)\right)\right] d t
\end{aligned}
$$

for all $(x, y) \in\left[x_{0}, X\right] \times\left[y_{0}, y_{1}\right)$. Integrating (2.27) from $x_{0}$ to $x$, by the definition of $W$ in (2.2), we get

$$
\begin{aligned}
W(\Xi(x, y)) \leq & W\left(\Xi\left(x_{0}, y\right)\right)+b(X, y) \sum_{i=1}^{n} \int_{\alpha_{i}\left(x_{0}\right)}^{\alpha_{i}(x)} \int_{\beta_{i}\left(y_{0}\right)}^{\beta_{i}(y)}\left[f_{i}(s, t) \varphi_{1}\left(\psi^{-1}(\Xi(s, t))\right)\right. \\
& \left.+g_{i}(s, t) \varphi_{2}\left(\log \left(\psi^{-1}(\Xi(s, t))\right)\right)\right] d t d s \\
= & W(a(X, y))+b(X, y) \sum_{i=1}^{n} \int_{\alpha_{i}\left(x_{0}\right)}^{\alpha_{i}(x)} \int_{\beta_{i}\left(\gamma_{0}\right)}^{\beta_{i}(y)}\left[f_{i}(s, t) \varphi_{1}\left(\psi^{-1}(\Xi(s, t))\right)\right. \\
& \left.+g_{i}(s, t) \varphi_{2}\left(\log \left(\psi^{-1}(\Xi(s, t))\right)\right)\right] d t d s,
\end{aligned}
$$


for all $(x, y) \in\left[x_{0}, X\right] \times\left[y_{0}, y_{1}\right)$.

When $\varphi_{1}(u) \geq \varphi_{2}(\log (u))$, from the inequality (2.28), we have

$$
\begin{aligned}
W(\Xi(x, y)) \leq & W(a(X, y))+b(X, y) \sum_{i=1}^{n} \int_{\alpha_{i}\left(x_{0}\right)}^{\alpha_{i}(x)} \int_{\beta_{i}\left(y_{0}\right)}^{\beta_{i}(y)}\left[f_{i}(s, t)\right. \\
& \left.+g_{i}(s, t)\right] \varphi_{1}\left(\psi^{-1}(\Xi(s, t))\right) d t d s,
\end{aligned}
$$

for all $(x, y) \in\left[x_{0}, X\right] \times\left[y_{0}, y_{1}\right)$. Now, define a function $\Theta(x, y)$ by the right-hand side of (2.29). Clearly, $\Theta(x, y)$ is a positive and nondecreasing function in each variable, $\Theta\left(x_{0}, y\right)$ $=W(a(X, y))>0$. Then, (2.29) is equivalent to

$$
\Xi(x, y) \leq W^{-1}(\Theta(x, y)), \quad \forall(x, y) \in\left[x_{0}, X\right] \times\left[y_{0}, Y_{2}\right),
$$

where $Y_{2}$ is defined by (2.23). Differentiating $\Theta(x, y)$ in $x$ for any fixed $y \in\left[y_{0}, Y_{2}\right)$, we have

$$
\begin{aligned}
\Theta_{x}(x, y) & =b(X, y) \sum_{i=1}^{n} \alpha_{i}^{\prime}(x) \int_{\beta_{i}\left(y_{0}\right)}^{\beta_{i}(y)}\left[f_{i}\left(\alpha_{i}(x), t\right)+g_{i}\left(\alpha_{i}(x), t\right)\right] \varphi_{1}\left(\psi^{-1}\left(\Xi\left(\alpha_{i}(x), t\right)\right)\right) d t \\
& \leq b(X, y) \varphi_{1}\left(\psi^{-1}\left(W^{-1}(\Theta(x, y))\right)\right) \sum_{i=1}^{n} \alpha_{i}^{\prime}(x) \int_{\beta_{i}\left(y_{0}\right)}^{\beta_{i}(y)}\left[f_{i}\left(\alpha_{i}(x), t\right)+g_{i}\left(\alpha_{i}(x), t\right)\right] d t,
\end{aligned}
$$

for all $(x, y) \in\left[x_{0}, X\right] \times\left[y_{0}, Y_{2}\right)$. From $(2.31)$, we have

$$
\frac{\Theta_{x}(x, y)}{\varphi_{1}\left(\psi^{-1}\left(W^{-1}(\Theta(x, y))\right)\right)} \leq b(X, y) \sum_{i=1}^{n} \alpha_{i}{ }^{\prime}(x) \int_{\beta_{i}\left(y_{0}\right)}^{\beta_{i}(y)}\left[f_{i}\left(\alpha_{i}(x), t\right)+g_{i}\left(\alpha_{i}(x), t\right)\right] d t
$$

for all $(x, y) \in\left[x_{0}, X\right] \times\left[y_{0}, Y_{2}\right)$. Integrating (2.32) from $x_{0}$ to $x$, by the definition of $\Psi_{1}$ in (2.22), we obtain

$$
\begin{aligned}
\Psi_{1}(\Theta(x, y)) & \leq \Psi_{1}\left(\Theta\left(x_{0}, y\right)\right)+b(X, y) \sum_{i=1}^{n} \int_{\alpha_{i}\left(x_{0}\right)}^{\alpha_{i}(x)} \int_{\beta_{i}\left(y_{0}\right)}^{\beta_{i}(y)}\left[f_{i}(s, t)+g_{i}(s, t)\right] d t d s \\
& =\Psi_{1}(W(a(X, y)))+b(X, y) \sum_{i=1}^{n} \int_{\alpha_{i}\left(x_{0}\right)}^{\alpha_{i}(x)} \int_{\beta_{i}\left(y_{0}\right)}^{\beta_{i}(y)}\left[f_{i}(s, t)+g_{i}(s, t)\right] d t d s .
\end{aligned}
$$

From (2.30) and (2.33), we conclude

$$
\begin{aligned}
u(x, y) \leq \psi^{-1}(\Xi(x, y)) \leq \psi^{-1}\left(W^{-1}(\Theta(x, y))\right) \leq \psi^{-1}\left[W ^ { - 1 } \left(\Psi_{1}^{-1}(\right.\right. \\
\left.\left.\Psi_{1}(W(a(X, y)))+b(X, y) \sum_{i=1}^{n} \int_{\alpha_{i}(x)}^{\alpha_{i}(x)} \int_{\beta_{i}\left(y_{0}\right)}^{\beta_{i}(y)}\left[f_{i}(s, t)+g_{i}(s, t)\right] d t d s\right)\right]
\end{aligned}
$$

for all $(x, y) \in\left[x_{0}, X\right] \times\left[y_{0}, Y_{2}\right)$. Let $x=X$, from (2.34), we get

$$
\begin{aligned}
& u(X, \gamma) \leq \psi^{-1}\left[W ^ { - 1 } \left(\Psi _ { 1 } ^ { - 1 } \left(\Psi_{1}(W(a(X, \gamma)))\right.\right.\right. \\
&\left.\left.+b(X, \gamma) \sum_{i=1}^{n} \int_{\alpha_{i}\left(x_{0}\right)}^{\alpha_{i}(X)} \int_{\beta_{i}\left(y_{0}\right)}^{\beta_{i}(y)}\left[f_{i}(s, t)+g_{i}(s, t)\right] d t d s\right)\right] .
\end{aligned}
$$


Since $X \in\left[x_{0}, X_{2}\right)$ is arbitrary, from the inequality (2.35), we obtain the required inequality in (2.20).

When $\varphi_{1}(u) \leq \varphi_{2}(\log (u))$, from the inequality (2.28), we have

$$
\begin{aligned}
W(\Xi(x, y)) \leq & W(a(X, y))+b(X, y) \sum_{i=1}^{n} \int_{\alpha_{i}\left(x_{0}\right)}^{\alpha_{i}(x)} \int_{\beta_{i}\left(y_{0}\right)}^{\beta_{i}(y)}\left[f_{i}(s, t)\right. \\
& \left.+g_{i}(s, t)\right] \varphi_{2}\left(\log \left(\psi^{-1}(\Xi(s, t))\right)\right) d t d s, \\
\leq & W(a(X, y))+b(X, y) \sum_{i=1}^{n} \int_{\alpha_{i}\left(x_{0}\right)}^{\alpha_{i}(x)} \int_{\beta_{i}\left(y_{0}\right)}^{\beta_{i}(\gamma)}\left[f_{i}(s, t)\right. \\
& \left.+g_{i}(s, t)\right] \varphi_{2}\left(\psi^{-1}(\Xi(s, t))\right) d t d s,
\end{aligned}
$$

for all $(x, y) \in\left[x_{0}, X\right] \times\left[y_{0}, y_{1}\right)$, where $x_{0} \leq X \leq X_{3}$. Similarly to the above process from (2.29) to (2.35), from (2.36), we obtain

$$
\begin{aligned}
& u(X, y) \leq \psi^{-1} {\left[W ^ { - 1 } \left(\Psi _ { 2 } ^ { - 1 } \left(\Psi_{2}(W(a(X, y)))\right.\right.\right.} \\
&\left.\left.+b(X, y) \sum_{i=1}^{n} \int_{\alpha_{i}\left(x_{0}\right)}^{\alpha_{i}(X)} \int_{\beta_{i}\left(y_{0}\right)}^{\beta_{i}(\gamma)}\left[f_{i}(s, t)+g_{i}(s, t)\right] d t d s\right)\right]
\end{aligned}
$$

Since $X \in\left[x_{0}, X_{3}\right)$ is arbitrary, where $X_{3}$ is defined by (2.24), from the inequality (2.37), we obtain the required inequality in (2.21).

Theorem 3. Suppose that $\left(H_{1}-H_{5}\right)$ hold and that $L, M \in C\left(\mathbb{R}_{+}^{3}, \mathbb{R}_{+}\right)$satisfy

$$
0 \leq L(s, t, u)-L(s, t, v) \leq M(s, t, v)(u-v)
$$

for $s, t, u, v \in \mathbb{R}_{+}$with $u>v \geq 0$. If $u(x, y)$ is a nonnegative and continuous function on $\Delta$ satisfying (1.3), then we have

$$
u(x, y) \leq \psi^{-1}\left[W^{-1}\left(\Psi_{3}^{-1}(E(x, y))\right)\right]
$$

for all $(x, y) \in\left[x_{0}, X_{4}\right) \times\left[y_{0}, Y_{4}\right)$, where $W$ is defined by $(2.2)$,

$$
\begin{aligned}
& \Psi_{3}(r):=\int_{1}^{r} \frac{d s}{\psi^{-1}\left(W^{-1}(s)\right)}, \quad r>0, \quad \Psi_{3}(0):=\lim _{r \rightarrow 0+} \Psi_{3}(r), \\
& E(x, y):=\Psi_{3}(F(x, y))+b(x, y) \sum_{i=1}^{n} \int_{i=1}^{\alpha_{i}(x)} \int_{\beta_{i}\left(y_{0}\right)}^{\beta_{i}(\gamma)}\left[f_{i}(s, t) M(s, t, 0)+g_{i}(s, t)\right] d t d s, \\
& F(x, y):=W(a(x, y))+b(x, y) \sum_{i=1}^{n} \int_{\alpha_{i}\left(x_{0}\right)}^{\alpha_{i}(x)} \int_{\beta_{i}\left(y_{0}\right)}^{\beta_{i}(y)} f_{i}(s, t) L(s, t, 0) d t d s,
\end{aligned}
$$

$\psi^{-1}, W^{-1}$ and $\Psi_{3}^{-1}$ denote the inverse function of $\psi, W$ and $\Psi_{3}$, respectively, and $\left(X_{4}, Y_{4}\right)$ $\in \Delta$ is arbitrarily given on the boundary of the planar region 


$$
\mathcal{R}:=\left\{(x, y) \in \Delta: E(x, y) \in \operatorname{Dom}\left(\Psi_{3}^{-1}\right), \Psi_{3}^{-1}(E(x, y)) \in \operatorname{Dom}\left(W^{-1}\right)\right\} .
$$

Proof. From the inequality (1.3), we have

$$
\begin{aligned}
\psi(u(x, y)) \leq & a(X, y)+b(X, y) \sum_{i=1}^{n} \int_{\alpha_{i}(x 0)}^{\alpha_{i}(x)} \int_{\beta_{i}\left(y_{0}\right)}^{\beta_{i}(y)} w(u(s, t))\left[f_{i}(s, t) L(s, t, u(s, t))\right. \\
& \left.+g_{i}(s, t) u(s, t)\right] d t d s,
\end{aligned}
$$

for all $(x, y) \in\left[x_{0}, X\right] \times\left[y_{0}, y_{1}\right)$, where $x_{0} \leq X \leq X_{4}$ is chosen arbitrarily. Let $P(x, y)$ denote the right-hand side of (2.42), which is a positive and nondecreasing function in each variable, $P\left(x_{0}, y\right)=a(X, y)$. Similarly to the process in the proof of Theorem 2 from (2.25) to (2.28), we obtain

$$
\begin{aligned}
W(P(x, y)) \leq W & (a(X, y))+b(X, y) \sum_{i=1}^{n} \int_{\alpha_{i}\left(x_{0}\right)}^{\alpha_{i}(x)} \int_{\beta_{i}\left(y_{0}\right)}^{\beta_{i}(y)}\left[f_{i}(s, t) L\left(s, t, \psi^{-1}(P(s, t))\right)\right. \\
+ & \left.g_{i}(s, t) \psi^{-1}(P(s, t))\right] d t d s, \quad \forall(x, y) \in\left[x_{0} X\right] \times\left[y_{0}, \gamma_{1}\right) .
\end{aligned}
$$

From the inequality (2.38) and (2.43), we get

$$
\begin{aligned}
W(P(x, y)) \leq & W(a(X, y))+b(X, y) \sum_{i=1}^{n} \int_{\alpha_{i}\left(x_{0}\right)}^{\alpha_{i}(X)} \int_{\beta_{i}\left(y_{0}\right)}^{\beta_{i}(y)} f_{i}(s, t) L(s, t, 0) d t d s \\
& +b(X, y) \sum_{i=1}^{n} \int_{\alpha_{i}\left(x_{0}\right)}^{\alpha_{i}(x)} \int_{\beta_{i}\left(y_{0}\right)}^{\beta_{i}(y)}\left[f_{i}(s, t) M(s, t, 0)+g_{i}(s, t)\right] \psi^{-1}(P(s, t)) d t d s,
\end{aligned}
$$

for all $(x, y) \in\left[x_{0}, X\right] \times\left[y_{0}, y_{1}\right)$. Similarly to the process in the proof of Theorem 2 from (2.29) to (2.35), we obtain

$$
\begin{aligned}
u(X, y) \leq & \psi^{-1}\left[W ^ { - 1 } \left(\Psi _ { 3 } ^ { - 1 } \left(\Psi_{3}\left(W(a(X, y))+b(X, y) \sum_{i=1}^{n} \int_{\alpha_{i}\left(x_{0}\right)}^{\alpha_{i}(X)} \int_{\beta_{i}\left(y_{0}\right)}^{\beta_{i}(y)} f_{i}(s, t) L(s, t, 0) d t d s\right)\right.\right.\right. \\
& \left.\left.+b(X, \gamma) \sum_{i=1}^{n} \int_{\alpha_{i}\left(x_{0}\right)}^{\alpha_{i}(X)} \int_{\beta_{i}\left(y_{0}\right)}^{\beta_{i}(y)}\left[f_{i}(s, t) M(s, t, 0)+g_{i}(s, t)\right] d t d s\right)\right],
\end{aligned}
$$

where $\Psi_{3}$ is defined by (2.40). Since $X \in\left[x_{0}, X_{4}\right)$ is arbitrary, where $X_{4}$ is defined by (2.41), from the inequality (2.44), we obtain the required inequality in (2.39).

\section{Applications to BVP}

In this section we use our result to study certain properties of solution of the following boundary value problem (simply called BVP):

$$
\left\{\begin{array}{l}
\frac{\partial^{2} \psi(z(x, y))}{\partial x \partial y}=F\left(x, y, z\left(\alpha_{1}(x), \beta_{1}(y)\right), z\left(\alpha_{2}(x), \beta_{2}(y)\right), \ldots, z\left(\alpha_{n}(x), \beta_{n}(y)\right)\right) \\
z\left(x, y_{0}\right)=a_{1}(x), \quad z\left(x_{0}, y\right)=a_{2}(y), a_{1}\left(x_{0}\right)=a_{2}\left(y_{0}\right)=0
\end{array}\right.
$$

for $x \in I, y \in J$, where $x_{0}, y_{0}, x_{1}, y_{1} \in \mathbb{R}_{+}$are constants, $I:=\left[x_{0}, x_{1}\right), J:=\left[y_{0}, y_{1}\right), F \in C(I \times$ $\left.J \times \mathbb{R}^{n}, \mathbb{R}\right), \psi: \mathbb{R} \rightarrow \mathbb{R}$ is strictly increasing on $\mathbb{R}_{+}$with $\psi(0)=0,|\psi(r)|=\psi(|r|)>0$, for $\mid$ 
$r \mid>0$ and $\psi(t) \rightarrow \infty$ as $t \rightarrow \infty$; functions $\alpha_{i} \in C^{1}(I, I) ; \beta_{i} \in C^{1}(J, J), i=1,2, \ldots, n$ are nondecreasing such that $\alpha_{i}(x) \leq x, \beta_{i}(y) \leq y, \alpha_{i}\left(x_{0}\right)=x_{0}, \beta_{i}\left(y_{0}\right)=y_{0} ;\left|a_{1}\right| \in C^{1}\left(I, \mathbb{R}_{+}\right),\left|a_{2}\right| \in C^{1}$ $\left(J, \mathbb{R}_{+}\right)$are both nondecreasing. Though this equation is similar to the equation discussed in Section 3 in [3], our results are more general than the results obtained in [3].

We first give an estimate for solutions of the BVP (3.1) so as to obtain a condition for boundedness.

Corollary 1. Consider BVP (3.1) and suppose that $F \in C\left(I \times J \times \mathbb{R}^{n}, \mathbb{R}\right)$ satisfies

$$
\left|F\left(x, y, u_{1}, u_{2}, \ldots, u_{n}\right)\right| \leq \sum_{i=1}^{n} w\left(\left|u_{i}\right|\right)\left[f_{i}(x, y) \varphi\left(\left|u_{i}\right|\right)+g_{i}(x, y)\right], \quad(x, y) \in I \times J,
$$

where $f_{i}, g_{i} \in C\left(I \times J, \mathbb{R}_{+}\right)$and $w, \varphi \in C\left(\mathbb{R}_{+}, \mathbb{R}_{+}\right)$are nondecreasing such that $w(u)>0, \varphi$ $(u)>0$ for $u>0$. Then all solutions $z(x, y)$ of $B V P(3.1)$ have the estimate

$$
|z(x, y)| \leq \psi^{-1}\left(W^{-1}\left(\Phi^{-1}(B(x, y))\right)\right),
$$

for all $(x, y) \in\left[x_{0}, X_{1}\right) \times\left[y_{0}, Y_{1}\right)$, where

$$
\begin{aligned}
& B(x, y):=\Phi(A(x, y))+\sum_{i=1}^{n} \int_{\alpha_{i}\left(x_{0}\right)}^{\alpha_{i}(x)} \int_{\beta_{i}\left(y_{0}\right)}^{\beta_{i}(y)} \frac{f_{i}\left(\alpha_{i}^{-1}(s), \beta_{i}^{-1}(t)\right)}{\alpha_{i}^{\prime}\left(\alpha_{i}^{-1}(s)\right) \beta_{i}^{\prime}\left(\beta_{i}^{-1}(t)\right)} d t d s, \\
& A(x, y):=W\left(\psi\left(\left|a_{1}(x)\right|\right)+\psi\left(\left|a_{2}(y)\right|\right)\right)+\sum_{i=1}^{n} \int_{\alpha_{i}\left(x_{0}\right)}^{\alpha_{i}(x)} \int_{\beta_{i}\left(\gamma_{0}\right)}^{\beta_{i}^{-1}} \frac{g_{i}\left(\alpha_{i}^{-1}(s), \beta_{i}^{-1}(t)\right)}{\alpha_{i}^{\prime}\left(\alpha_{i}^{-1}(s)\right) \beta_{i}^{\prime}\left(\beta_{i}^{-1}(t)\right)} d t d s,
\end{aligned}
$$

for all $(x, y) \in\left[x_{0}, X_{1}\right) \times\left[y_{0}, Y_{1}\right)$, where functions $W, W^{-1}, \Phi, \Phi^{-1}$ and real numbers $X_{1}$, $Y_{1}$ are given as in Theorem 1.

Proof. The equivalent integral equation of BVP (3.1) is

$$
\begin{aligned}
\psi(z(x, y))= & \psi\left(a_{1}(x)\right)+\psi\left(a_{2}(y)\right)+\int_{x_{0}}^{x} \int_{\gamma_{0}}^{\gamma} F\left(s, t, z\left(\alpha_{1}(s), \beta_{1}(t)\right), z\left(\alpha_{2}(s), \beta_{2}(t)\right), \ldots,\right. \\
& \left.z\left(\alpha_{n}(s), \beta_{n}(t)\right)\right) d t d s .
\end{aligned}
$$

By (3.2) and (3.4), we get that

$$
\begin{aligned}
\psi & (|z(x, y)|) \\
\leq & \psi\left(\left|a_{1}(x)\right|\right)+\psi\left(\left|a_{2}(y)\right|\right) \\
& +\int_{x_{0}}^{x} \int_{y_{0}}^{y}\left|F\left(s, t, z\left(\alpha_{1}(s), \beta_{1}(t)\right), z\left(\alpha_{2}(s), \beta_{2}(t)\right), \ldots, z\left(\alpha_{2}(s), \beta_{n}(t)\right)\right)\right| d t d s \\
\leq & \left(\left|a_{1}(x)\right|\right)+\psi\left(\left|a_{2}(y)\right|\right) \\
& +\int_{x_{0}}^{x} \int_{y_{0}}^{y} \sum_{i=1}^{n} w\left(\left|z\left(\alpha_{i}(s), \beta_{i}(t)\right)\right|\right)\left[f_{i}(s, t) \varphi\left(\left|z\left(\alpha_{i}(s), \beta_{i}(t)\right)\right|\right)+g_{i}(s, t)\right] d t d s \\
= & \psi\left(\left|a_{1}(x)\right|\right)+\psi\left(\left|a_{2}(y)\right|\right)+\sum_{i=1}^{n} \int_{\alpha_{i}\left(x_{0}\right)}^{\alpha_{i}(x)} \int_{\beta_{i}\left(y_{0}\right)}^{\beta_{i}(y)} \\
& \frac{w\left(\left|z\left(s_{1}, t_{1}\right)\right|\right)\left[f_{i}\left(\alpha_{i}^{-1}\left(s_{1}\right), \beta_{i}^{-1}\left(t_{1}\right)\right) \varphi\left(\left|z\left(s_{1}, t_{1}\right)\right|\right)+g_{i}\left(\alpha_{i}^{-1}\left(s_{1}\right), \beta_{i}^{-1}\left(t_{1}\right)\right)\right]}{\alpha_{i}{ }^{\prime}\left(\alpha_{i}^{-1}\left(s_{1}\right)\right) \beta_{i}{ }^{\prime}\left(\beta_{i}^{-1}\left(t_{1}\right)\right)} d t_{1} d s_{1},
\end{aligned}
$$


where a change of variables $s_{1}=\alpha_{i}(s), t_{1}=\beta_{i}(t), i=1,2, \ldots, n$ are made. Clearly, the inequality (3.5) is in the form of (1.1). Thus the estimate (3.3) of the solution $z(x, y)$ in this corollary is obtained immediately by our Theorem 1 .

Our Corollary 1 actually gives a condition of boundedness for solutions. Concretely, if

$$
\begin{aligned}
& \psi\left(\left|a_{1}(x)\right|\right)+\psi\left(\left|a_{2}(y)\right|\right)<\infty, \\
& \sum_{i=1}^{n} \int_{\alpha_{i}\left(x_{0}\right)}^{\alpha_{i}(x)} \int_{\beta_{i}\left(y_{0}\right)}^{\beta_{i}(y)} \frac{f_{i}\left(\alpha_{i}^{-1}(s), \beta_{i}^{-1}(t)\right)}{\alpha_{i}{ }^{\prime}\left(\alpha_{i}^{-1}(s)\right) \beta_{i}^{\prime}\left(\beta_{i}^{-1}(t)\right)} d t d s<\infty, \\
& \sum_{i=1}^{n} \int_{\alpha_{i}\left(x_{0}\right)}^{\alpha_{i}(x)} \int_{\beta_{i}\left(y_{0}\right)}^{\beta_{i}(y)} \frac{g_{i}\left(\alpha_{i}^{-1}(s), \beta_{i}^{-1}(t)\right)}{\alpha_{i}{ }^{\prime}\left(\alpha_{i}^{-1}(s)\right) \beta_{i}{ }^{\prime}\left(\beta_{i}^{-1}(t)\right)} d t d s<\infty,
\end{aligned}
$$

on $\left[x_{0}, X_{1}\right) \times\left[y_{0}, Y_{1}\right)$, then every solution $z(x, y)$ of BVP (3.1) is bounded on $\left[x_{0}, X_{1}\right) \times$ $\left[y_{0}, Y_{1}\right)$.

Next, we discuss the uniqueness of solutions for BVP (3.1).

Corollary 2. Consider BVP (3.1) and suppose that $F \in C\left(I \times J \times \mathbb{R}^{n}, \mathbb{R}\right)$ satisfies

$$
\left|F\left(x, y, u_{1}, u_{2}, \ldots, u_{n}\right)-F\left(x, y, v_{1}, v_{2}, \ldots, v_{n}\right)\right| \leq \sum_{i=1}^{n} f_{i}(x, y)\left|\psi\left(u_{i}\right)-\psi\left(v_{i}\right)\right|
$$

for all $(x, y) \in I \times J$ and $u_{i}, v_{i} \in \mathbb{R}, i=1,2, \ldots, n$, where $I=\left[x_{0}, x_{1}\right], J=\left[y_{0}, y_{1}\right]$ are two finite intervals, and $f_{i} \in C\left(I \times J, \mathbb{R}_{+}\right), i=1,2, \ldots, n$. Then $B V P$ (3.1) has at most one solution on $I \times J$.

Proof. Assume that both $z(x, y)$ and $\tilde{z}(x, y)$ are solutions of BVP (3.1). From the equivalent integral Equations (3.4) and (3.6), we have

$$
\begin{aligned}
& |\psi(z(x, y))-\psi(\tilde{z}(x, y))| \\
& \leq \int_{x_{0}}^{x} \int_{y_{0}}^{\gamma} \mid F\left(s, t, z\left(\alpha_{1}(s), \beta_{1}(t)\right), z\left(\alpha_{2}(s), \beta_{2}(t)\right), \ldots, z\left(\alpha_{n}(s), \beta_{n}(t)\right)\right) \\
& \quad-F\left(s, t, \tilde{z}\left(\alpha_{1}(s), \beta_{1}(t)\right), \tilde{z}\left(\alpha_{2}(s), \beta_{2}(t)\right), \ldots, \tilde{z}\left(\alpha_{n}(s), \beta_{n}(t)\right)\right) \mid d t d s \\
& \leq \int_{x_{0}}^{x} \int_{\gamma_{0}}^{\gamma} \sum_{i=1}^{n} f_{i}(s, t)\left|\psi\left(z\left(\alpha_{i}(s), \beta_{i}(t)\right)\right)-\psi\left(\tilde{z}\left(\alpha_{i}(s), \beta_{i}(t)\right)\right)\right| d t d s \\
& \leq \varepsilon+\int_{\alpha_{i}\left(x_{0}\right)}^{\alpha_{i}(x)} \int_{\beta_{i}\left(y_{0}\right)}^{\beta_{i}(\gamma)} \sum_{i=1}^{n} \frac{f_{i}\left(\alpha_{i}^{-1}\left(s_{1}\right), \beta_{i}^{-1}\left(t_{1}\right)\right)\left|\psi\left(z\left(s_{1}, t_{1}\right)\right)-\psi\left(\tilde{z}\left(s_{1}, t_{1}\right)\right)\right|}{\alpha_{i}{ }^{\prime}\left(\alpha_{i}^{-1}\left(s_{1}\right)\right) \beta_{i}^{\prime}\left(\beta_{i}^{-1}\left(t_{1}\right)\right)} d t_{1} d s_{1},
\end{aligned}
$$

for all $(x, y) \in I \times J$, where changes of variables $s_{1}=\alpha_{i}(s), t_{1}=\beta_{i}(t)$ are made, $\varepsilon>0$ is an arbitrary small number. Clearly, the inequality (3.7) is in the form of (1.1). Suitably applying our Theorem 1 to (3.7), we get an estimate of the form (2.1) for all $(x, y) \in I \times J$,

$$
|\psi(z(x, y))-\psi(\tilde{z}(x, y))| \leq \varepsilon \exp \left(\int_{\alpha_{i}\left(x_{0}\right)}^{\alpha_{i}(x)} \int_{\beta_{i}\left(y_{0}\right)}^{\beta_{i}(y)} \sum_{i=1}^{n} \frac{f_{i}\left(\alpha_{i}^{-1}(s), \beta_{i}^{-1}(t)\right)}{\alpha_{i}^{\prime}\left(\alpha_{i}^{-1}(s)\right) \beta_{i}^{\prime}\left(\beta_{i}^{-1}(t)\right)} d t d s\right) .
$$


Letting $\varepsilon \rightarrow 0_{+}$, since $\int_{\alpha_{i}\left(x_{0}\right)}^{\alpha_{i}(x)} \int_{\beta_{i}\left(y_{0}\right)}^{\beta_{i}(y)} \sum_{i=1}^{n} \frac{f_{i}\left(\alpha_{i}^{-1}(s), \beta_{i}^{-1}(t)\right)}{\alpha_{i}^{\prime}\left(\alpha_{i}^{-1}(s)\right) \beta_{i}^{\prime}\left(\beta_{i}^{-1}(t)\right)} d t d s$ is finite on finite intervals $I$ and $J, \psi$ is a strictly increasing function, from (3.8), we conclude that $|\psi(z(x, y))-\psi(\tilde{z}(x, y))| \leq 0$, implying that $z(x, y)=\tilde{z}(x, y)$ for all $(x, y) \in I \times J$. The uniqueness is proved.

Remark Suppose that $F \in C\left(I \times J \times \mathbb{R}^{n}, \mathbb{R}\right)$ in BVP (3.1) satisfies

$$
\begin{aligned}
\left|F\left(x, y, u_{1}, u_{2}, \ldots, u_{n}\right)\right| \leq & \sum_{i=1}^{n} \int_{\alpha_{i}\left(x_{0}\right)}^{\alpha_{i}(x)} \int_{\beta_{i}\left(y_{0}\right)}^{\beta_{i}(y)} w(u(s, t))\left[f_{i}(s, t) \varphi_{1}(u(s, t))\right. \\
& \left.+g_{i}(s, t) \varphi_{2}(\log (u(s, t)))\right] d t d s .
\end{aligned}
$$

By using Theorem 2, we can give an estimate for solutions of the BVP (3.1).

Suppose that $F \in C\left(I \times J \times \mathbb{R}^{n}, \mathbb{R}\right)$ in BVP (3.1) satisfies

$$
\begin{aligned}
\left|F\left(x, y, u_{1}, u_{2}, \ldots, u_{n}\right)\right| \leq & \sum_{i=1}^{n} \int_{\alpha_{i}\left(x_{0}\right)}^{\alpha_{i}(x)} \int_{\beta_{i}\left(y_{0}\right)}^{\beta_{i}(y)} w(u(s, t))\left[f_{i}(s, t) L(s, t, u(s, t))\right. \\
& \left.+g_{i}(s, t) u(s, t)\right] d t d s .
\end{aligned}
$$

By using Theorem 3, we can give an estimate for solutions of the BVP (3.1) too.

\begin{abstract}
Acknowledgements
The authors are very grateful to the editor and the referees for their helpful comments and valuable suggestions. This research was supported by the National Natural Science Foundation of China (Project No. 11161018), Guangxi Natural Science Foundation (Project No. 0991265), the Key Project of Hechi University (2009YAZ-N001) and the Key Discipline of Applied Mathematics of Hechi University of China(200725).
\end{abstract}

\title{
Competing interests
}

The author declares that they have no competing interests.

Received: 13 October 2011 Accepted: 15 February 2012 Published: 15 February 2012

\section{References}

1. Bellman, R: The stability of solutions of linear differential equations. Duke Math J. 10, 643-647 (1943). doi:10.1215/S00127094-43-01059-2

2. Gronwall, TH: Note on the derivatives with respect to a parameter of the solutions of a system of differential equations. Ann Math. 20, 292-296 (1919). doi:10.2307/1967124

3. Agarwal, RP, Kim, YH, Sen, SK: New retarded integral inequalities with applications. J Inequal Appl 2008, 15 (2008). (Article ID 908784)

4. Bainov, D, Simeonov, P: Integral Inequalities and Applications. Kluwer Academic, Dordrecht (1992)

5. Bihari, IA: A generalization of a lemma of Bellman and its application to uniqueness problem of differential equation. Acta Math Acad Sci Hung. 7, 81-94 (1956). doi:10.1007/BF02022967

6. Chen, CJ, Cheung, WS, Zhao, D: Gronwall-Bellman-Type integral inequalities and applications to BVPS. J Inequal Appl 2009, 15 (2009). (Article ID 258569)

7. Cheung, WS: Some new nonlinear inequalities and applications to boundary value problems. Nonlinear Anal. 64, 2112-2128 (2006). doi:10.1016/j.na.2005.08.009

8. Choi, SK, Deng, S, Koo, NJ, Zhang, W: Nonlinear integral inequalities of Bihari-Type without class H. Math Inequal Appl. 8(4), 643-654 (2005)

9. Dafermos, CM: The second law of thermodynamics and stability. Arch Rat Mech Anal. 70, 167-179 (1979)

10. Dannan, F: Integral inequalities of Gronwall-Bellman-Bihari type and asymptotic behavior of certain second order nonlinear differential equations. J Math Anal Appl. 108, 151-164 (1985). doi:10.1016/0022-247X(85)90014-9

11. Dragomir, SS, Kim, YH: Some integral inequalities for functions of two variables. Electron J Diff Equ. 2003(10), 1-13 (2003)

12. Lipovan, O: A retarded Gronwall-like inequality and its applications. J Math Anal Appl. 252, 389-401 (2000). doi:10.1006/ jmaa.2000.7085

13. $\mathrm{Ma}, \mathrm{QH}$, Yang, EH: Some new Gronwall-Bellman-Bihari type integral inequalities with delay. Periodica Mathematica Hungarica. 44, 225-238 (2002). doi:10.1023/A:1019600715281

14. Massalitina, EV: On the Perow integro-summable inequality for functions of two variables. Ukrainian Math J. 56, 1864-1872 (2004). doi:10.1007/s11253-005-0156-0 
15. Medina, R, Pinto, M: On the asymptotic behavior of solutions of a class of second order nonlinear differential equations. J Math Anal Appl. 135, 399-405 (1988). doi:10.1016/0022-247X(88)90163-1

16. Mitrinović, DS, Pečarić, JE, Fink, AM: Inequalities Involving Functions and Their Integrals and Derivatives. Kluwer Academic, Dordrecht (1991)

17. Pachpatte, BG: On some new inequalities related to certain inequalities in the theory of differential equations. J Math Anal Appl. 189, 128-144 (1995). doi:10.1006/jmaa.1995.1008

18. Pachpatte, BG: Inequalities for Differential and Integral Equations. Academic Press Limited, London (1998)

19. Pachpatte, BG: On some new nonlinear retarded integral inequalities. J Inequal Pure Appl Math 5(3), 8 (2004). (Article ID 80)

20. Pinto, M: Integral inequalities of Bihari-type and applications. Funkcial Ekvac. 33, 387-430 (1990)

21. Wang, WS: A generalized retarded Gronwall-like inequality in two variables and applications to BVP. Appl Math Comput. 191, 144-154 (2007). doi:10.1016/j.amc.2007.02.099

22. Wang, WS, Shen, CX: On a generalized retarded integral inequality with two variables. J Inequal Appl 2008, 9 (2008). (Article ID 518646)

23. Wang, WS: Some new nonlinear Gronwall-Bellman-Type integral inequalities and applications to BVPs. J Appl Math Comput. (2010)

doi:10.1186/1029-242X-2012-31

Cite this article as: Wang: Some generalized nonlinear retarded integral inequalities with applications. Journal of Inequalities and Applications 2012 2012:31.

\section{Submit your manuscript to a SpringerOpen ${ }^{\circ}$} journal and benefit from:

- Convenient online submission

- Rigorous peer review

- Immediate publication on acceptance

- Open access: articles freely available online

- High visibility within the field

- Retaining the copyright to your article

Submit your next manuscript at $\gg$ springeropen.com 